\title{
Correction to: Mutations in RHOT1 Disrupt Endoplasmic Reticulum-Mitochondria Contact Sites Interfering with Calcium Homeostasis and Mitochondrial Dynamics in Parkinson's Disease by Grossmann et al. Antioxid. Redox Signal. 31: 1213-1234, 2019. DOI: 10.1089/ars.2018.7718
}

N the December 1, 2019 issue of Antioxidants \& Redox Signaling (vol. 31, no. 16, 1213-1234) the article entitled "Mutations in RHOT1 Disrupt Endoplasmic Reticulum-Mitochondria Contact Sites Interfering with Calcium Homeostasis and Mitochondrial Dynamics in Parkinson's Disease" by Grossmann et al. requires correction.

In Figure 2I, the microscopy image of control fibroblasts is not representative of the results shown in Figure $2 \mathrm{H}$. The related microscopy image for the control in Figure 2I was erroneously chosen from another control cell line, not part of the study. The original Figure 2I is shown below:
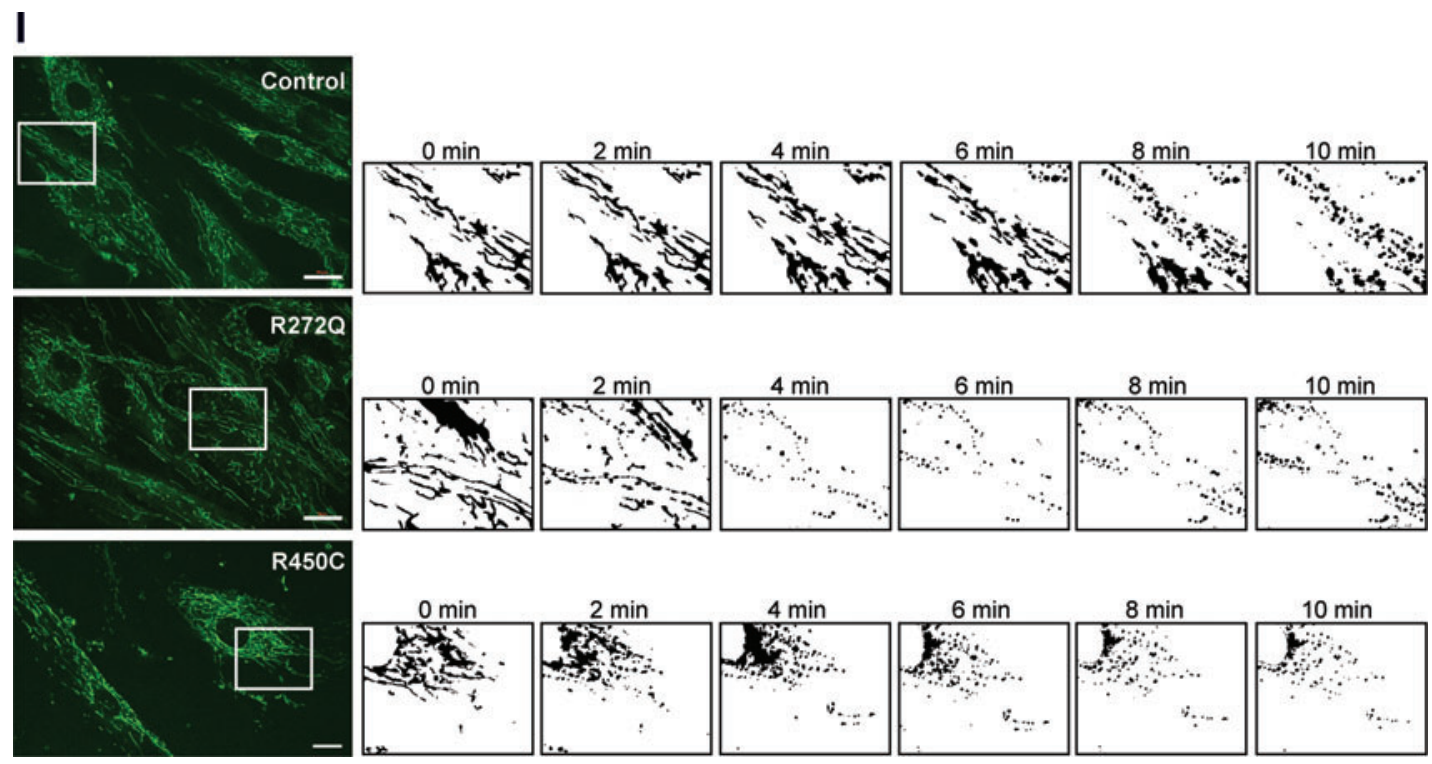

Color images are available online. 
Figure 2I has been replaced with the correct image:
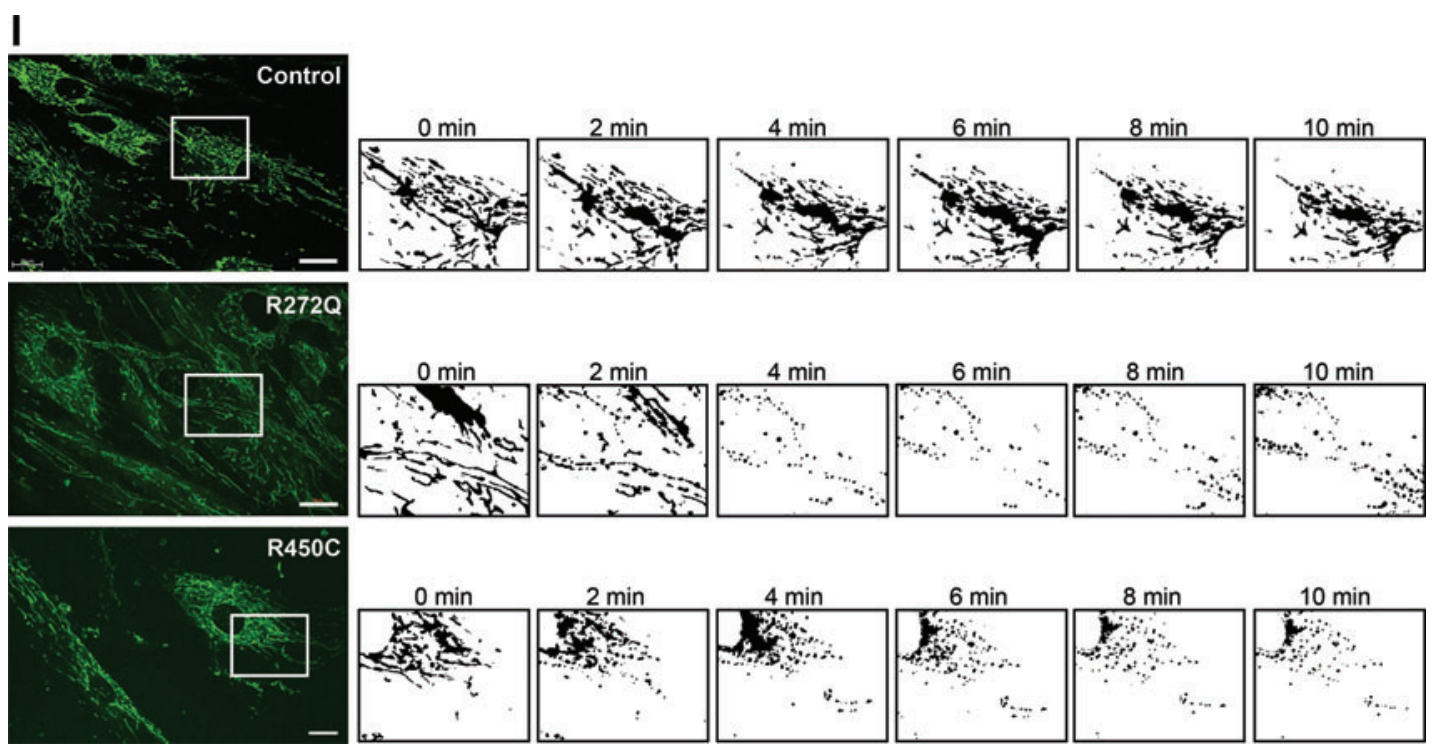

Color images are available online.

The online version has been corrected to reflect this. The authors apologize for this error. 